\title{
Atuação dos profissionais de saúde e o processo de humanização no centro obstétrico
}

RESUMO | Objetivo: Verificar na literatura científica a atuação dos profissionais de saúde acerca do processo de humanização no centro obstétrico. Método: Trata-se de um estudo bibliográfico, tipo revisão sistemática. Realizou-se a busca por artigos; publicados nos últimos cinco anos (2014 a 2019); nos idiomas português, inglês e espanhol; disponíveis na íntegra. Foram consultadas as seguintes plataformas de dados: BDENF, MEDLINE, LILACS e SciELO. Resultado: Inicialmente foram encontrados 174; contudo, após a leitura permaneceram apenas os que atendiam aos critérios para inclusão e exclusão descritos na metodologia, totalizando 10 estudos. Conclusão: Observou-se a imprescindibilidade de reformar as práticas diárias, consolidar a adoção de práticas firmadas em fundamentos científicos impulsionando os profissionais e a sociedade civil a assegurar as gestantes um atendimento de saúde com igualdade e remediabilidade; e livre de violência obstétrica.

Palavras-chaves: Pessoal de Saúde; Parto Humanizado; Salas de Parto.

ABSTRACT | Objective: To verify in the scientific literature the performance of health professionals regarding the humanization process in the obstetric center. Method: This is a bibliographic study, type systematic review. The search for articles was carried out; published in the last five years (2014 to 2019); in Portuguese, English and Spanish; available in full. The following data platforms were consulted: BDENF, MEDLINE, LILACS and SciELO. Result: Initially 174 were found; however, after reading, only those who met the inclusion and exclusion criteria described in the methodology remained, totaling 10 studies. Conclusion: It was observed that the indispensability of reforming daily practices, consolidating the adoption of practices based on scientific foundations, boosting professionals and civil society to ensure that pregnant women receive health care with equality and remediation; and free from obstetric violence.

Keywords: Health Personnel; Humanizing Delivery; Delivery Rooms.

RESUMEN | Objetivo: Verificar en la literatura científica el desempeño de los profesionales de la salud con respecto al proceso de humanización en el centro obstétrico. Método: Este es un estudio bibliográfico, tipo revisión sistemática. Se realizó la búsqueda de artículos; publicado en los últimos cinco años (2014 a 2019); en portugués, inglés y español; Disponible en su totalidad. Se consultaron las siguientes plataformas de datos: BDENF, MEDLINE, LILACS y SciELO. Resultado: inicialmente se encontraron 174; sin embargo, después de leer, solo aquellos que cumplieron con los criterios de inclusión y exclusión descritos en la metodología permanecieron, totalizando 10 estudios. Conclusión: Se observó que la necesidad de reformar las prácticas diarias, consolidar la adopción de prácticas basadas en fundamentos científicos, impulsar a los profesionales y sociedad civil para asegurar que las mujeres embarazadas reciban atención médica con igualdad y remediación; y libre de violencia obstétrica.

Palavras claves: Personal de Salud; Parto Humanizado; Salas de Parto.

\section{Ana Cecilia Fragoso Veloso}

Especialista em Enfermagem em Centro Cirúrgico, Sala Anestésica e Central de Material e Esterilização (CME) pela Faculdade Redentor - IDE. Recife, Pernambuco (PE), Brasil.

ORCID: 0000-0002-4791-9693

\section{Liniker Scolfild Rodrigues da Silva}

Especialista em Enfermagem Obstétrica na modalidade Residência pela Faculdade de Enfermagem Nossa Senhora das Graças/ Universidade de Pernambuco (FENSG/ UPE). Sanitarista, Especialista em Saúde Coletiva na modalidade Residência pela Faculdade de Ciências Médicas (FCM)/UPE. Recife, Pernambuco (PE), Brasil.

ORCID: 0000-0003-3710-851X

\section{Phelipe Gomes de Barros}

Doutorando em Ciências pela Faculdade de Medicina da Universidade de São Paulo (USP). Recife, Pernambuco (PE), Brasil.

ORCID: 0000-0003-2568-4034

\section{Roberta Rosas Trigueiro Gomes}

Especialista em Enfermagem em Centro Cirúrgico, Sala Anestésica e Central de Material e Esterilização (CME) pela Faculdade Redentor - IDE. Recife, Pernambuco (PE), Brasil.

ORCID: 0000-0002-4341-3441

\section{Auridéa Souza dos Santos}

Especialista em Enfermagem Obstétrica pela Faculdade Metropolitana. Recife, Pernambuco (PE), Brasil.

ORCID: 0000-0001-9090-1301

\section{Helena Maria da Silva Oliveira}

Enfermeira graduada pela Faculdade São Miguel. Recife, Pernambuco (PE), Brasil. ORCID: 0000-0001-5632-7940

Recebido em: 22/07/2020

Aprovado em: 31/07/2020
INTRODUÇÃO

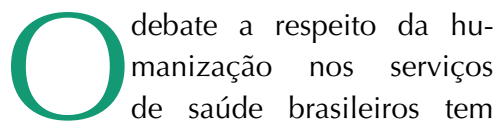
provocado transformações nas práticas profissionais, associadas aos princípios do Sistema Único de Saúde (SUS), que buscam a integralidade da assistência e à equidade no acesso e na participação social do usuário, oportunizando alterações no ambiente de trabalho que estimem as relações entre usuários e profissionais ${ }^{1}$.

No mundo, inúmeras mulheres são vítimas de violência obstétrica enquanto estão em trabalho de parto nas instituições de saúde. O termo "violência obstétrica" reúne e relata diversas formas de violência e danos provocados pelo profissional de saúde durante a assistência no pré-natal, parto, puerpério e abortamento. Desta maneira, pode ser determinada como maus-tratos físicos, 
psicológicos e verbais, ou como práticas intervencionistas desnecessárias ${ }^{2}$.

No ano de 2014, a Organização Mundial da Saúde (OMS) declarou que todas as mulheres têm o direito de receber o mais alto nível de assistência médica, o que inclui o direito a cuidados dignos e respeitosos na gravidez e no parto. e o direito de não sofrer violência ou discriminação. Na qual, foi solicitado um maior apoio dos governos em relação ao apoio a pesquisas e ações destinadas a melhorar o tratamento prestado às mulheres durante o parto institucionalizado ${ }^{3}$.

Diante desta perspectiva, a Política Nacional de Humanização (PNH) qualifica a atenção obstétrica e se consolida como política transversal no SUS, colaborando com práticas de trabalho em concordância com o divulgado nas normatizações que a sustentam. Como política de Estado, deve se preocupar com a formação dos profissionais que seguirão suas diretrizes e, primordialmente, estimular a inserção da humanização na formação dos profissionais para o SUS ${ }^{1}$.

A equipe de saúde tem papel fundamental na implementação do parto humanizado. Segundo a OMS, a gestação de baixo risco pode ser acompanhada por um enfermeiro obstétrico, sendo este também
“
Diante desta perspectiva, a Política Nacional de Humanização (PNH) qualifica a atenção obstétrica

e se consolida como política transversal no SUS, colaborando com práticas de trabalho em concordância com o divulgado nas normatizações que a sustentam.

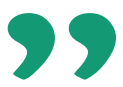

Figura 1: Definição da questão norteadora de acordo com a estratégia de PICO, Recife, Pernambuco (PE), Brasil, 2020.

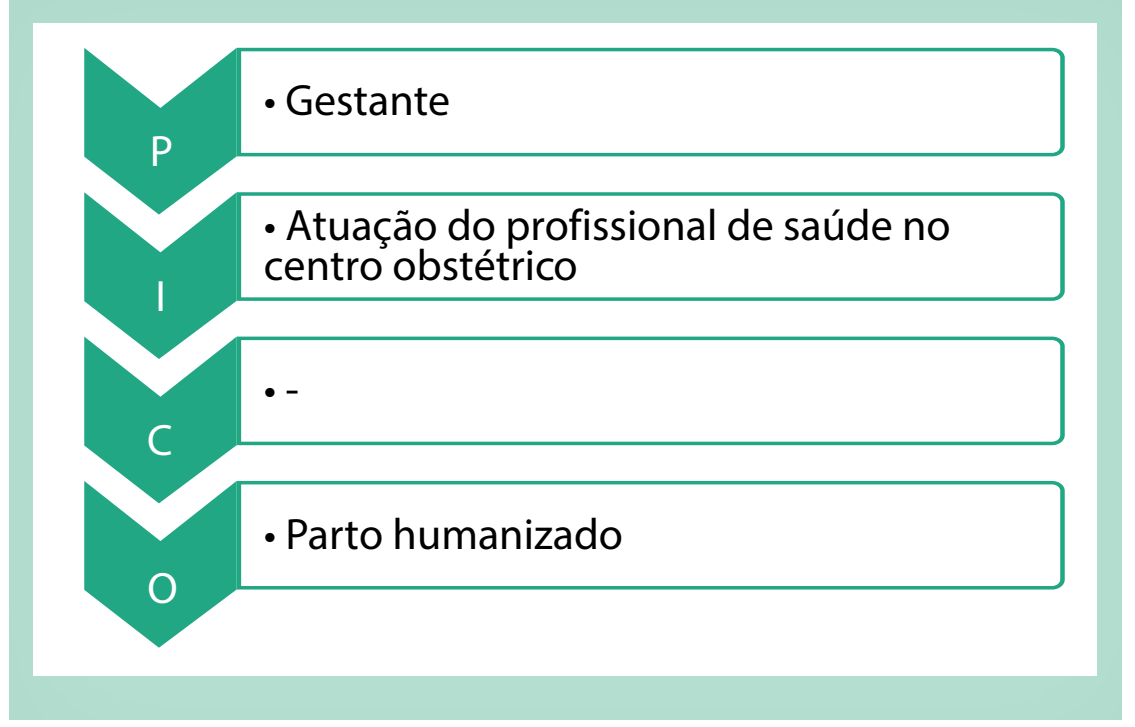

Fonte: Elaboração própria. qualificado. A implementação de Programas de Residência Multiprofissionais e em Área Profissional da Saúde, estabelecidas pela Portaria Interministerial no 1.077 , de 12 de novembro de 2009, são estabelecidas como instrumento fundamental para o alcance das metas propostas pelo MS para atenção ao parto e nascimento ${ }^{2,4,5}$.

Portanto, diante desse cenário, é relevante analisar a atuação dos profissionais de saúde que atuam nos centros de assistência obstétrica, com intuito de identificar como é realizado o processo de humanização, que afeta diretamente na qualidade de vida e assistência prestada. Os resultados poderão subsidiar a reflexão dos desafios e impasses para a mudança desse panorama no âmbito da Enfermagem. Este estudo tem como objetivo verificar na literatura científica a atuação dos profissionais de saúde acerca do processo de humanização no centro obstétrico.

\section{MÉTODO}

Trata-se de um estudo bibliográfico, tipo revisão integrativa com abordagem qualitativa e quantitativa, onde realizou-se a busca de artigos para desenvolvimento metodológico e foram percorridas, as seguintes etapas: elaboração da questão norteadora e objetivo do estudo; definição de critérios de inclusão e exclusão das produções científicas; busca de estudos científicos nas bases de dados e bibliotecas virtuais; análise e categorização das produções encontradas; resultados e discussão dos achados ${ }^{6}$.

Para o levantamento da questão norteadora, aplicou-se a estratégia PICO (P-população/paciente, I- Intervenção/interesse, C- Comparação/Ausência e O -Desfecho) onde $\mathrm{C}$ permaneceu ausente, pois não houveram elementos de cunho comparativo. Estratégia baseada na segmentação da hipótese, que visa o levantamento de dados de forma sistematizada ${ }^{7}$.

Desta forma, definiu-se a seguinte questão norteadora da pesquisa: "Como os profissionais de saúde que atuam no centro obstétrico realizam o processo de humanização?" (Figura 1). 
Figura 2: Fluxograma da seleção dos estudos segundo o Preferred Reporting Items for Systematic Reviews and Meta-Analyses (PRISMA 2015). Recife, Pernambuco (PE), Brasil, 2020.

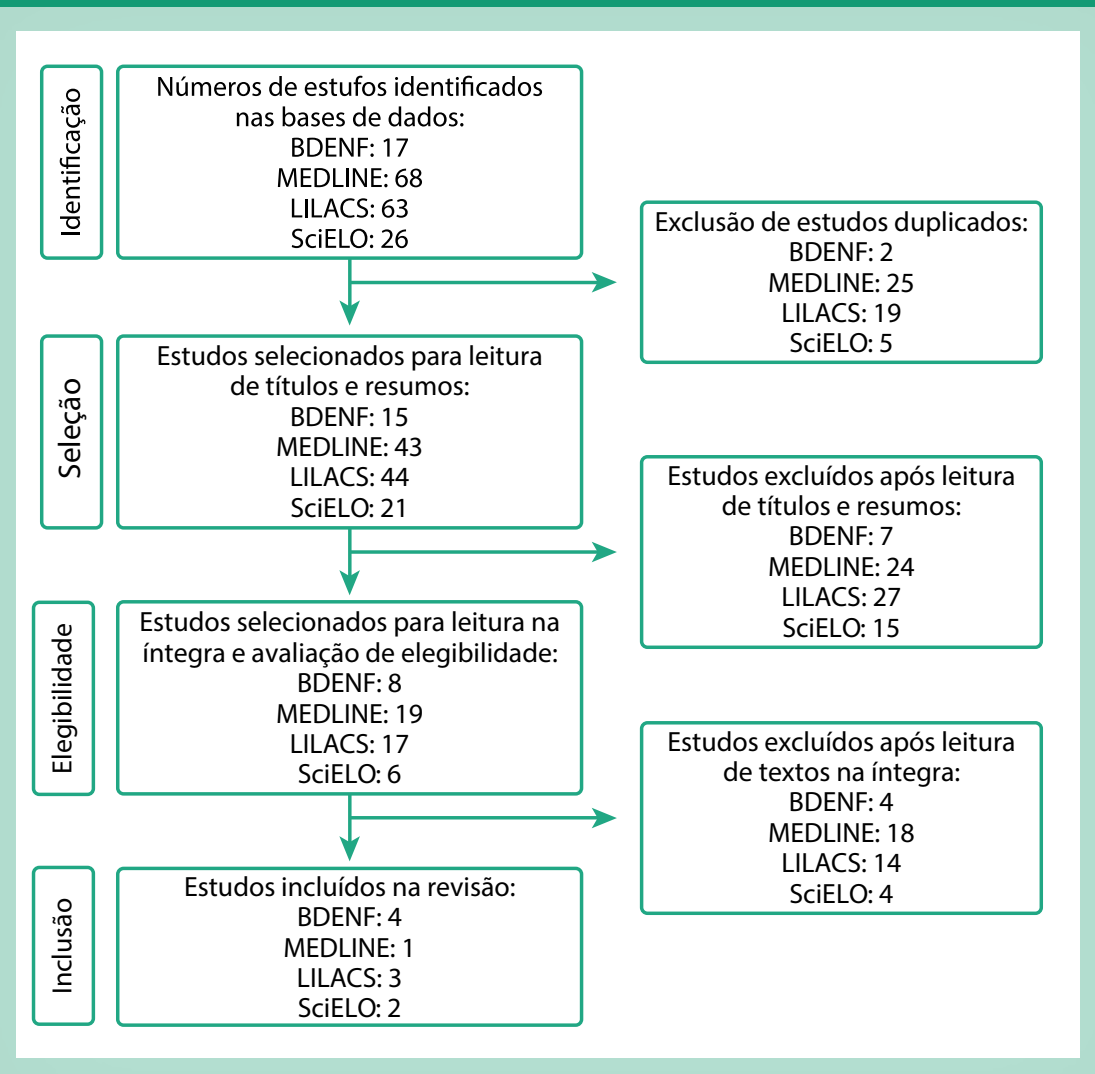

Fonte: Elaboração própria.

Tabela 1: Publicações selecionadas acerca do tema, organizadas conforme as Bases de dados/Biblioteca virtual.Recife, Pernambuco (PE), Brasil, 2020.

\begin{tabular}{cccc}
$\begin{array}{c}\text { Base de Dados/ } \\
\text { Biblioteca Virtual }\end{array}$ & $\begin{array}{c}\text { Artigos } \\
\text { Encontrados }\end{array}$ & $\begin{array}{c}\text { Artigos } \\
\text { Selecionados para } \\
\text { Leitura na íntegra }\end{array}$ & Amostra Final \\
\hline BDENF & 17 & 8 & 4 \\
\hline MEDLINE & 68 & 19 & 1 \\
\hline LILACS & 63 & 17 & 3 \\
\hline SciELO & 26 & 6 & 2 \\
\hline
\end{tabular}

Tabela 2: Resultados encontrados nos estudos de acordo com título, base de dados, autores, ano de publicação, delineamento, níveis de evidencia, local e idioma. Recife, Pernambuco (PE), Brasil, 2020.

Título/Base de Dados

Contentamento de

A puérperas assistidas por enfermeiros obstetras / BDENF.
Autor/Ano

Delineamento

Local/Idioma

Ribeiro et al., (2018)

Estudo exploratório, descritivo de abordagem quantitativa.
Para seleção dos artigos, utilizaram-se como critérios de inclusão: ser artigo original, ter sido publicado em português, inglês ou espanhol, nos últimos cinco anos (2014 a 2019), disponível na íntegra. Foram excluídos teses, dissertações e monografias, editoriais, estudos de caso, as revisões integrativas, sistemáticas e conceituais, estudos repetidos, além de artigos que não responderam à questão norteadora.

O levantamento dos dados, que ocorreu durante os meses de abril e maio de 2020 nas seguintes bases de dados e bibliotecas virtuais: Base de Dados de Enfermagem (BDENF), Medical Literature Analysis and Retrieval System Online (MEDLINE); na Literatura Latino-Americana e do Caribe em Ciências da Saúde (LILACS); e na Biblioteca Virtual Scientific Electronic Library Online (SciELO). Foram realizados cruzamentos dos descritores "Pessoal da saúde", "Parto humanizado", "Sala de Parto" presentes na base de Descritores em Ciências da Saúde (DecS) combinados com operador booleano AND e OR.

A princípio eliminaram-se por meio da leitura de títulos e resumos, estudos duplicados. Destes pré-selecionados, realizou-se leitura na íntegra, a fim de verificar os que atendem à questão norteadora e aos critérios de inclusão/exclusão. Construiu-se então a amostra final com estudos pertinentes aos critérios pré-estabelecidos (Figura 2).

A seleção dos estudos, baseou-se no Preferred Reporting Items for Systematic Reviewand Meta-Analyse (PRISMA)um checklist com 27 itens e um fluxograma de quatro etapas, com o objetivo de auxiliar no desenvolvimento de artigos e o nível de evidência dos estudos selecionados foi determinado de acordo com a Agency for Healthcare Research and Quality8,9. Visando simplificar o entendimento das publicações selecionadas nesta revisão integrativa, organizaram-se os dados em figuras e tabelas, expostos de forma descritiva. 


\begin{tabular}{|c|c|c|c|c|}
\hline B & $\begin{array}{c}\text { Fatores associados } \\
\text { à humanização da } \\
\text { assistência em uma } \\
\text { maternidade pública / } \\
\text { BDENF. }\end{array}$ & $\begin{array}{l}\text { Inagaki et al., } \\
\text { (2018) }\end{array}$ & $\begin{array}{l}\text { Estudo quanti-qua- } \\
\text { litativo, transversal, } \\
\text { descritivo. }\end{array}$ & $\begin{array}{c}\text { Brasil (Pernambuco) } \\
\text { /Português. }\end{array}$ \\
\hline C & $\begin{array}{l}\text { Interfaces entre } \\
\text { profissionais de saúde } \\
\text { e a humanização da } \\
\text { assistência ao parto / } \\
\text { BDENF. }\end{array}$ & $\begin{array}{l}\text { Pinto et al., } \\
\text { (2018) }\end{array}$ & $\begin{array}{c}\text { Estudo descritivo de } \\
\text { abordagem qualitati- } \\
\text { va. }\end{array}$ & $\begin{array}{l}\text { Brasil (Distrito Fede- } \\
\text { ral) / Português. }\end{array}$ \\
\hline D & $\begin{array}{l}\text { Contato pele a pele do } \\
\text { recém-nascido com sua } \\
\text { mãe na perspectiva da } \\
\text { equipe multiprofissio- } \\
\text { nal/ BDENF. }\end{array}$ & $\begin{array}{l}\text { Kologeski et } \\
\text { al., (2017) }\end{array}$ & $\begin{array}{l}\text { Estudo exploratório, } \\
\text { descritivo de aborda- } \\
\text { gem quantitativa. }\end{array}$ & $\begin{array}{c}\text { Brasil (Pernambuco) } \\
\text { /Português. }\end{array}$ \\
\hline$E$ & $\begin{array}{l}\text { Uso de práticas integra- } \\
\text { tivas e complementares } \\
\text { por doulas em mater- } \\
\text { nidades de Fortaleza } \\
\text { (CE) e Campinas (SP) / } \\
\text { LILACS. }\end{array}$ & $\begin{array}{l}\text { Silva et al., } \\
\text { (2016) }\end{array}$ & Estudo qualitativo. & $\begin{array}{l}\text { Brasil (São Paulo) / } \\
\text { Português. }\end{array}$ \\
\hline
\end{tabular}

No parto vaginal e na cesariana acompanhan-

$F$ te não entra: discursos de enfermeiras e diretores técnicos / LILACS.

\begin{tabular}{|c|c|c|c|c|}
\hline G & $\begin{array}{c}\text { A equipe de saúde e a } \\
\text { segurança do binômio } \\
\text { mãe-bebê no parto e } \\
\text { nascimento / LILACS. }\end{array}$ & $\begin{array}{c}\text { Dornfeld e } \\
\text { Pedro. (2015) }\end{array}$ & $\begin{array}{l}\text { Estudo exploratório, } \\
\text { descritivo de aborda- } \\
\text { gem qualitativa. }\end{array}$ & $\begin{array}{c}\text { Brasil (Rio Grande } \\
\text { do Sul) / Inglês. }\end{array}$ \\
\hline
\end{tabular}

Negociação de padrões de qualidade para 0

H fornecimento efetivo de cuidados de parto e parto na Nigéria e Uganda / MEDLINE.

\begin{tabular}{|c|c|c|c|c|}
\hline I & $\begin{array}{c}\text { Práticas de atendi- } \\
\text { mento do parto numa } \\
\text { instituição prestadora } \\
\text { de serviços da saúde } \\
\text { na cidade de Bogotá / } \\
\text { SciELO. }\end{array}$ & $\begin{array}{l}\text { Rocha-Acero; } \\
\text { Socarrás- } \\
\text {-Ronderos e } \\
\text { Rubio-León. } \\
\text { (2019) }\end{array}$ & $\begin{array}{l}\text { Estudo interpreta- } \\
\text { tivo, descritivo de } \\
\text { abordagem qualitati- } \\
\text { va. }\end{array}$ & Colômbia/ Espanhol. \\
\hline
\end{tabular}

Baixa luminosidade em J sala de parto: vivências de enfermeiras obstétricas / SciELO.

\section{Oladapo et \\ al., (2017)}

Rodrigues e

Shimo. (2019)
Estudo exploratório, descritivo de abordagem qualitativa.

Brasil (Rio Grande do Sul) /Português. ções selecionadas conforme as bases de dados analisadas.

$\mathrm{Na}$ tabela 2, os estudos levantados estão dispostos destacando, seus títulos, autores, anos de publicação, delineamento, local e idioma. Onde pode-se observar que a maior parte dos estudos era nacional, publicados em português $(n=7)$, dois artigos eram internacionais um publicado em inglês e espanhol.

\section{DISCUSSÃO}

Após a leitura dos estudos selecionados, os artigos foram categorizados em recortes temáticos, classificando o conhecimento produzido acerca do tema, em níveis de evidência, majoritariamente nível IV- Estudo com delineamento não-experimental como pesquisa descritiva correlacional e qualitativa ou estudos de caso $^{9}$.

A humanização do parto busca a superação do medo e do isolamento que as mulheres sofrem no modelo assistencial obstétrico hegemônico, medicalizado e intervencionista. O enfermeiro tem a educação em saúde como uma das atribuições convenientes a sua profissão. É essencial desmistificar a cultura da cesárea e ressaltar para as gestantes os benefícios de um parto normal e sem interferências, quando possível, para que esta possa decidir com mais consciência sobre o método a ser selecionado ${ }^{4,10}$.

Segundo a OMS a gestação de baixo risco pode ser assistida por um enfermeiro obstétrico e que a sua atuação no trabalho de parto, proporciona satisfação à parturiente e sua família e também ao profissional. Um estudo relatou que a humanização, essencialmente, exige do enfermeiro uma visão humanística e a necessidade de entender o outro, e para que isso ocorra de forma completa, é preciso, que seja realizada a troca de informações e de sentimentos entre essas pessoas, de maneira empática ${ }^{11}$.
LO; contudo, após a leitura permaneceram apenas os que atendiam aos critérios para inclusão e exclusão descritos na metodologia, totalizando 10 estudos. Destaca-se na tabela 1 as publicaestavam disponíveis na BDENF; 68 na MEDLINE; 63 na LILACS; e 26 na SciE-
Estudo exploratório, gem qualitativa.
Brasil (Rio Grande do Sul) /Português.
Fonte: Elaboração própria.

\section{RESULTADOS}

Dos 174 estudos encontrados, 17

$\begin{array}{ccc}\begin{array}{c}\text { Brüggemann } \\ \text { et al., (2015) }\end{array} & \begin{array}{c}\text { descritivo de aborda- } \\ \text { gem qualitativa. }\end{array} & \begin{array}{c}\text { Brasil (Rio Grande } \\ \text { do Sul) /Português. }\end{array}\end{array}$


Todos os profissionais que atuam na assistência ao parto, sejam médicos, enfermeiros, técnicos e auxiliares de enfermagem e mesmo os funcionários administrativos, necessitam ser qualificados e sensibilizados quanto à humanização do cuidado, inclusive com assuntos associados ao acolhimento. É necessário observar os cuidados fisiológicos e emocionais da parturiente, proporcionando uma experiência boa, minimizando a insegurança e as angústias ${ }^{12}$.

Considerando-se a presença de indícios científicos que evidenciam crescentemente as vantagens de uma assistência humanizada, é essencial que a formação acadêmica integre as transformações no padrão de atenção à saúde da mulher de maneira integral. Desta forma, é necessário que ocorra a assimilação de novas condutas por parte dos profissionais, que certifique a saúde e os direitos das mulheres e de seus recém-nascidos; além da efetiva incorporação do modelo humanizado de atenção como diretriz e filosofia institucional busca respeitar a fisiologia da mulher e favorecer os interesses de todos ${ }^{13}$.

\section{CONCLUSÃO}

Este estudo proporcionou identificação de várias publicações científicas acerca do processo de humanização da assistência ao parto e análise. A enfermagem surge como a conexão entre esses atores, estando sua essência ligada ao cuidado. É um profissional indispensável para o desenvolvimento da humanização, dentro deste cenário.

Constata-se que inúmeras políticas de humanização da assistência obstétrica têm sido desenvolvidas, também por solicitação da própria sociedade. A capacitação, atualização e inclusão do tema na graduação dos profissionais da saúde têm recebido atenção e investimentos.

Corroborando, observou-se a imprescindibilidade de reformar as práticas diárias, consolidar a adoção de práticas firmadas em fundamentos científicos e impulsionar os profissionais e a sociedade civil sobre a questão para conceituar o sistema e assegurar às gestantes um atendimento de saúde com igualdade e remediabilidade; e livre de violência obstétrica, que proporcione a fortificação e consolidação do SUS. 2

\section{Referências}

1. Ferreira júnior A.R., et al. Percepções de Profissionais de Enfermagem Sobre a Humanização em Obstetrícia. Sanare. 2015 jul;14(2):27-35.

2. Menezes F.R., et al. 0 olhar de residentes em Enfermagem Obstétrica para o contexto da violência obstétrica nas instituições.Interface[Internet]. 2019 Set[cited 2020 May 10];24 Availablefrom:http://www.scielo.br/scielo.php?script=sci_arttext\&pid=\$1414-32832020000100204\&Ing=en\&nrm=iso 3. WHO-Organização Mundial da Saúde [Internet]. Suíça: WHO; 2014. Prevenção e erradicação de desrespeito e abuso durante o parto em centros de saúde; [cited 2020 May 10]; Availablefrom: http://apps.who.int/iris/bitstream/handle/10665/134590/WHO_RHR_14.23_spa.pdf?sequence=1 4. Almeida O.S.C.; Gama E.R.; BahianaP.M. Humanização do Parto: a atuação dos enfermeiros. Revista Enfermagem Contemporânea. 2015 aug;4(1):79-90. 5. Jardim D.M.B., et al. Residência em enfermagem obstétrica no Hospital Sofia Feldman: uma estratégia de formação no e para o SUS. In: Ferla AA et al., organizadores. Residências e a educação e ensino da saúde: tecnologias formativas e o desenvolvimento do trabalho.Rede Unida.2017; 5(2):102-107. 6. Soares C.B.,et al. Integrative Review: ConceptsAndMethodsUsed In Nursing. Revista da Escola de Enfermagem da Usp. 2014 apr; 48(2):335-345. 7. Santos M.A.R.C., Galvão M.G.A. A elaboração da pergunta adequada de pesquisa. ResidPediatr. 2014 aug;4(2):53-56.
8. Galvão T.F., PansaniT.S.A., Harrad D. Principais itens para relatar Revisões sistemáticas e Meta-análises: A recomendação PRISMA. Epidemiologia e Serviços de Saúde, Brasília (Tradução para o idioma português do documento: Moher D, Liberati A, Tetzlaff J, Altman DG, The PRISMA Group. Preferred Reporting Items for Systematic Reviews and Meta-Analyses: The PRISMA Statement) [Internet]. 2015 jun [cited 2020 May 10];24(2):335-342.Availablefrom: ww.prisma-statement.org.

9. Agency For Health CareResearchAndQuality(Rockville)(Org.)[Internet].2016Qualitylmprovement and monitoring at your fingertips; 2016. [cited 2020 May 10]; Availablefrom: https://www.qualityindicators.ahrq.gov/ 10. Arruda S.S.,et al. Atuação da Enfermagem no Parto Humanizado. In: II CONGRESSO BRASILEIRO DE CIÊNCIAS DA SAUDE (Anais). 2017;2:1-8. 11. Melo A.A.P.,et al. Atuação do Enfermeiro no Parto Humanizado. Revista Científica Eletrônica de Enfermagem da Faef. 2018 jun; 1(1):4-11.

12. Alves M.A.C.B., Lippi U.G., GarciA S.A.L. Reflexões sobre a humanização na assistência de enfermagem obstétrica na visão do enfermeiro obstetra. Enfermagem Brasil. 2015 feb;14 (2):99-110.

13. Pereira R.M., et al. Novas práticas de atenção ao parto e os desafios para a humanização da assistência nas regiões sul e sudeste do Brasil. Ciência \& Saúde Coletiva. 2018 nov;23(11):3517-3524. 